\title{
SORÇÃO E DESSORÇÃO DO IMAZAQUIN EM SOLOS COM DIFERENTES CARACTERÍSTICAS GRANULOMÉTRICAS, QUÍMICAS E MINERALÓGICAS ${ }^{(1)}$
}

\author{
Robson Rolland Monticelli Barizon ${ }^{(2)}$, Arquimedes Lavorenti ${ }^{(3)}$, \\ Jussara Borges Regitano ${ }^{(4)}$ \& Valdemar Luiz Tornisielo ${ }^{(4)}$
}

\begin{abstract}
RESUMO
O presente estudo teve o objetivo de avaliar a sorção e dessorção do imazaquin em solos com diferentes características granulométricas, químicas e mineralógicas por meio de isotermas e estudos de cinética e, assim, quantificar a histerese no processo de sorção-dessorção. Os solos utilizados foram classificados como Latossolo Vermelho distroférrico (LVdf), Latossolo VermelhoAmarelo (LVA) e Neossolo Quartzarênico (RQ). Os solos foram secos ao ar e peneirados em malha de $2 \mathrm{~mm}$, sendo então procedida à caracterização dessas amostras. A molécula radiomarcada com ${ }^{14} \mathrm{C}$ utilizada foi o imazaquin, herbicida do grupo das imidazolinonas. A radioatividade foi determinada por espectrometria de cintilação líquida. Os ensaios foram realizados em sala climatizada $\left(25 \pm 2{ }^{\circ} \mathrm{C}\right)$. Nas isotermas de sorção, foram utilizadas cinco concentrações do imazaquin $\left(0,67 ; 1,34 ; 2,68 ; 5,36 ; 10,72 \mu \mathrm{mol} \mathrm{L} \mathrm{L}^{-1}\right)$, sendo os resultados ajustados à equação de Freundlich, obtendo, assim, os parâmetros de sorção. Na seqüência, foram realizadas quatro extrações com solução $0,005 \mathrm{~mol} \mathrm{~L}^{-1}$ de $\mathrm{CaCl}_{2}$, determinando-se os parâmetros de dessorção de forma similar à sorção. No ensaio de cinética de sorção, foi determinada a quantidade sorvida de imazaquin, a partir da adição de solução de $2,99 \mu \mathrm{mol} \mathrm{\textrm {L } ^ { - 1 }}$ do imazaquin, em períodos preestabelecidos $(0 ; 0,5 ; 1 ; 3 ; 6 ; 12 ; 24$ e $48 \mathrm{~h}$, após a aplicação do imazaquin), com os resultados sendo ajustados à equação de Elovich. De maneira geral, o imazaquin apresentou baixa sorção para os três solos, com os maiores coeficientes de sorção nos solos com maior teor de argila e CO. Os coeficientes
\end{abstract}

\footnotetext{
${ }^{(1)}$ Parte da Tese de Doutorado em Agronomia do primeiro autor. Programa de Pós-Graduação em Solos e Nutrição de Plantas, Escola Superior de Agricultura "Luiz de Queiroz" - ESALQ/USP. Trabalho financiado pela CAPES e FAPESP. Recebido para publicação em agosto de 2004 e aprovado em junho de 2005.

${ }^{(2)}$ Pesquisador do Bioagri Laboratórios Ltda. Rodovia SP 127, Km 24, Caixa Postal 573, CEP 13412-000 Piracicaba (SP). E-mail: r.barizon@bioagri.com.br

${ }^{(3)}$ Professor Titular do Departamento de Ciências Exatas, Escola Superior de Agricultura "Luiz de Queiroz" - ESALQ/USP. Av. Pádua Dias 11, CEP 13418-900 Piracicaba (SP). E-mail: alavoren@esalq.usp.br

${ }^{(4)}$ Pesquisador do Centro de Energia Nuclear na Agricultura - CENA/USP. Caixa Postal 96, CEP 13400-970 Piracicaba (SP). E-mail: vltornis@cena.usp.br; regitano@cena.usp.br
} 
de dessorção foram maiores que os coeficientes de sorção, demonstrando a ocorrência de histerese. A cinética de sorção mostrou que o imazaquin foi sorvido em duas etapas: uma rápida, que apresentou a maior fração sorvida, e outra lenta. A aplicação da Lei de Fick aos dados experimentais do ensaio de cinética indica que mecanismos de difusão podem estar envolvidos neste processo.

Termos de indexação: histerese, herbicida, isotermas, cinética.

\section{SUMMARY: IMAZAQUIN SORPTION AND DESORPTION IN SOILS WITH DIFFERENT MINERALOGICAL, PHYSICAL AND CHEMICAL CHARACTERISTICS}

The aim of this study was to evaluate the sorption and desorption of imazaquin in soils with different granulometric, chemical and mineralogical characteristics. Isotherms and kinetics studies were carried out to quantify the hysteresis between sorption and desorption processes. The soils were classified as Rhodic Hapludox (LVdf), Typic Hapludox (LVA) and Typic Quartzipsamment (RQ). The soils were air-dried and passed through a $2 \mathrm{~mm}$ mesh sieve, and the chemical, physical and mineralogical properties characterized. The radiolabeled molecule $\left({ }^{14} \mathrm{C}\right)$ was imazaquin, an herbicide of the imidazolinone group. Radioactivity was quantified by liquid scintillation spectrometry. The trials were carried out under controlled temperature conditions $\left(25 \pm 2^{\circ} \mathrm{C}\right)$. Five imazaquin concentrations were used $\left(0.67 ; 1.34 ; 2.68 ; 5.36\right.$; and $\left.10.72 \mu \mathrm{mol} \mathrm{L}^{-1}\right)$ for the sorption isotherms. The experimental data were fitted to the Freundlich equation so that sorption parameters were obtained. Subsequently, four extractions were performed with a $0.005 \mathrm{~mol} \mathrm{~L}^{-1} \mathrm{CaCl}_{2}$ solution in order to determine desorption parameters in a similar way for sorption. For sorption kinetics, the sorbed imazaquin amount $\left(2.99 \mu \mathrm{mol} \mathrm{L}^{-1}\right)$ was determined after different time intervals $(0 ; 0.5 ; 1 ; 3 ; 6 ; 12 ; 24$, and $48 \mathrm{~h}$ after imazaquin application), and the results adjusted to the Elovich equation. Imazaquin generally presented low sorption to the three soils, with higher sorption coefficients in the soils with higher clay and organic carbon content. The desorption coefficients were higher than those of sorption, evidencing the occurrence of hysteresis. Sorption kinetics showed that imazaquin is sorbed in two phases - a rapid one that presented the largest sorbed fraction, and another slower one. The application of Fick's Law to the kinetic data showed that diffusion mechanisms might be involved in this process.

Index terms: hysteresis, herbicide, icotherms, kinetics.

\section{INTRODUÇÃO}

Imazaquin, ácido 2-[4,5-dihidro-4-metil-4-(1metiletil)-5-oxo-1H-imidazol-2-il]-3-quinolinocarboxílico, é um herbicida sistêmico, pré-emergente, pertencente ao grupo químico das imidazolinonas, utilizado no controle de um amplo espectro de dicotiledôneas na cultura da soja (Bhalla et al., 1991). A molécula contém dois grupos funcionais ionizáveis: um grupo carboxílico (ácido, $\mathrm{pK}_{\mathrm{a}}=3,8$ ) e um grupo quinolina $\left(\mathrm{pK}_{\mathrm{a}}=2,0\right)$, o que lhe confere um caráter anfótero.

A sorção do imazaquin tem sido relacionada com os teores de argila e matéria orgânica no solo (Che et al., 1992; Regitano et al., 2000), apresentando baixa taxa de sorção nos solos mais arenosos com baixo teor de matéria orgânica. Esta característica do imazaquin, juntamente com sua persistência relativamente elevada (Basham \& Lavy, 1987), confere-lhe potencial de alta mobilidade no solo. Atualmente, com o advento de técnicas conservacionistas, como o sistema plantio direto, muitas áreas de solos arenosos têm sido incorporadas ao processo de produção agrícola para o cultivo de grãos, principalmente a soja. Assim, é de grande importância o estudo da sorção de pesticidas como o imazaquin em diversos cenários, incluindo os solos arenosos.

A baixa sorção do imazaquin deve-se, em grande parte, à repulsão causada por forças eletrostáticas. Estas forças repulsivas são geradas pela interação das cargas elétricas negativas da molécula de imazaquin e de cargas elétricas negativas dos colóides do solo. Em ambos os casos, o pH da solução do solo exerce relevante influência, seja pela dissociação dos grupos funcionais do imazaquin e de alguns componentes do solo, seja pela alteração das propriedades físico-químicas da molécula (Loux et al., 1989; Stougaard et al., 1990; Rocha et al., 2000). 
Grande parte dos processos e reações que ditam o destino de pesticidas no ambiente, como lixiviação, retenção e degradação, iniciam-se na solução do solo. Dentre as reações que determinam esta disponibilidade, a dessorção é de fundamental importância. Para compostos orgânicos, como os pesticidas, tem sido verificado que a energia de ligação na dessorção é maior que a energia de ligação na sorção, fenômeno conhecido como histerese (Zhu $\&$ Selim, 2000).

Parâmetros de dessorção para imazaquin são escassos na literatura. Che et al. (1992), avaliando sorção-dessorção de imazaquin e imazetapir, outro herbicida do grupo químico das imidazonilonas, constataram que o imazetapir dessorveu mais rapidamente que o imazaquin, sendo tal fato atribuído à maior afinidade do imazaquin às substâncias húmicas.

Muitos modelos têm sido propostos para explicar a histerese no processo de dessorção. Pignatello (2000) relacionou os seguintes mecanismos como prováveis causas da histerese: (a) formação de estados metaestáveis; (b) tempo de equilíbrio insuficiente para atingir o equilíbrio químico; (c) mudanças nas propriedades do sorvente, de tal forma que a dessorção ocorre num ambiente diferente daquele verificado na sorção. Esta última hipótese é mais relacionada com a matriz húmica do solo.

O objetivo deste estudo foi avaliar a sorção e dessorção do imazaquin em solos com diferentes características granulométricas, químicas e mineralógicas por meio de isotermas e estudos de cinética e, assim, quantificar a histerese no processo de sorção-dessorção.

\section{MATERIAL E MÉTODOS}

\section{Solo}

Foram utilizadas amostras deformadas de três solos: Latossolo Vermelho distroférrico (LVdf), Latossolo Vermelho-Amarelo (LVA) e Neossolo
Quartzarênico (RQ). Os três solos foram coletados no município de Piracicaba -SP. A cobertura vegetal no momento da coleta era cana-de-açúcar (LVA e LVdf) e pastagem (RQ). As amostras foram coletadas na camada superficial $(0-0,2 \mathrm{~m}$ de profundidade), secas ao ar e passadas em peneira com malha de $2 \mathrm{~mm}$. As características químicas foram deter-minadas de acordo com Raij \& Quaggio (1983). A análise granulométrica foi realizada de acordo com Camargo et al. (1986). O Fe total $\left(\mathrm{Fe}_{2} \mathrm{O}_{3}\right)$ e $\mathrm{Al}$ total $\left(\mathrm{Al}_{2} \mathrm{O}_{3}\right)$ foram extraídos com $\mathrm{H}_{2} \mathrm{SO}_{4}$ $18 \mathrm{~mol} \mathrm{~L}^{-1}$ (Vettori, 1969) e os minerais de argila predominantes foram identificados por difração de raios X (Jackson, 1969) (Quadro 1).

\section{Pesticida}

Foi utilizado o herbicida imazaquin: ácido 2-[4,5dihidro-4-metil-4-(1-metiletil)-5-oxo-1H-imidazol-2il]-3-quinolino-carboxílico. A molécula de imazaquin pertence ao grupo dos imidazolinonas, com baixa solubilidade em água $\left(\mathrm{S}_{\mathrm{w}}=60 \mathrm{mg} \mathrm{L} \mathrm{L}^{-1}, 25^{\circ} \mathrm{C} \mathrm{em}\right.$ $\mathrm{pH} 3,0$ ). Foi utilizada no experimento a molécula grau técnico (pureza $=98,2 \%$ ), bem como seu respectivo isótopo radioativo ${ }^{14} \mathrm{C}$-imazaquin (Figura 1), com marcação no grupo carboxílico (atividade específica $=0,80 \mathrm{MBq} \mathrm{mg}^{-1}$; pureza radioquímica $>98 \%$ ).

\section{Isotermas de sorção e dessorção}

O experimento de sorção/dessorção foi realizado com amostras dos três solos, em triplicata. Para o procedimento do estudo de isotermas de sorção, foram utilizadas cinco concentrações do imazaquin $(0,67 ; 1,34 ; 2,68 ; 5,36 ; 10,72 \mu \mathrm{mol} \mathrm{L}-1)$.

As cinco soluções de trabalho preparadas em $\mathrm{CaCl}_{2}$ 0,005 mol L-1 foram adicionadas $(10 \mathrm{~mL})$ às amostras de solo (5 g de solo seco). Após o tempo de equilíbrio (24 h), sob agitação constante (140 rpm) e centrifugação (10.000 rpm), foi coletada uma alíquota do sobrenadante $(1 \mathrm{~mL})$. A radioatividade no sobrenadante foi determinada por espectrometria de cintilação líquida (ELC), para o cálculo da concentração do pesticida sorvido. Considerou-se a quantidade de imazaquin sorvida como sendo a

Quadro 1. Características mineralógicas, químicas e granulométricas de um Neossolo Quartzarênico (RQ), um Latossolo Vermelho-Amarelo (LVA) e um Latossolo Vermelho distroférrico (LVdf)

\begin{tabular}{|c|c|c|c|c|c|c|c|c|c|}
\hline Solo & $p H_{H_{2}} \mathrm{o}$ & $\mathbf{p H}_{\mathrm{KCl}}$ & $\mathbf{C}_{\text {org }}$ & Areia & Silte & Argila & $\mathbf{A l}_{2} \mathbf{O}_{3}$ & $\mathrm{Fe}_{2} \mathrm{O}_{3}$ & Mineral de argila \\
\hline & & & $\mathrm{g} \mathrm{kg}^{-1}$ & 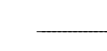 & $-\mathrm{g} \mathrm{kg}^{-1}$ & $\underline{-}$ & $\%$ & $\underline{-}$ & \\
\hline $\mathrm{RQ}$ & 5,9 & 4,9 & 5 & 900 & 20 & 80 & 2,29 & 0,34 & $\mathrm{Q}^{(1)}$ \\
\hline LVA & 6,1 & 5,3 & 11 & 700 & 100 & 200 & 5,22 & 2,88 & $\mathrm{C}^{(2)} ; \mathrm{Q}$ \\
\hline LVdf & 5,5 & 4,5 & 15 & 180 & 120 & 700 & 21,90 & 21,56 & $\mathrm{C} ; \mathrm{Q} ; \mathrm{Gb}^{(3)} ; \mathrm{M}^{(4)}$ \\
\hline
\end{tabular}

${ }^{(1)}$ Quartzo. ${ }^{(2)}$ Caulinita. ${ }^{(3)}$ Gibsita. ${ }^{(4)}$ Muscovita. 


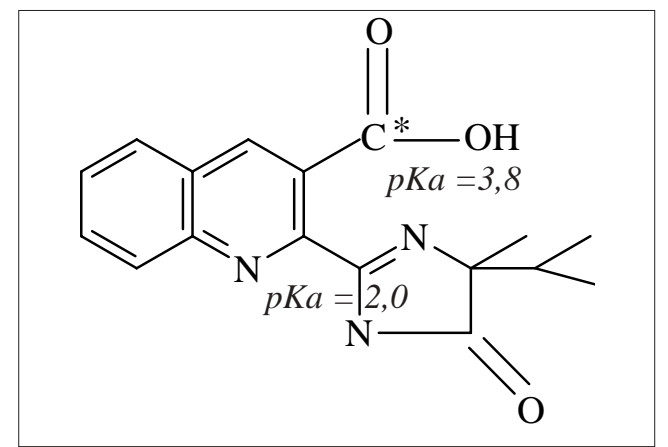

Figura 1. Estrutura química do imazaquin e localização da marcação do ${ }^{14} \mathrm{C}$.

diferença entre a concentração inicial do imazaquin na solução e a concentração presente na solução após decorrido o tempo de equilíbrio.

Os resultados de sorção foram ajustados à equação linearizada de Freundlich:

$$
\log \mathrm{S}=\log \mathrm{K}_{\mathrm{f}}+\mathrm{N} \log \mathrm{C}_{\mathrm{e}}
$$

na qual $\mathrm{S}$ = quantidade de imazaquin sorvida por unidade de massa de solo $(\mu \mathrm{mol} \mathrm{kg}-1), \mathrm{K}_{\mathrm{f}}=$ constante

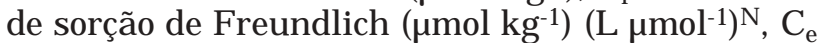
$=$ concentração de imazaquin na solução $\left(\mu \mathrm{mol} \mathrm{L} \mathrm{L}^{-1}\right)$ e $\mathrm{N}=$ declividade da isoterma. $\mathrm{O}$ coeficiente de sorção linear $\left(\mathrm{K}_{\mathrm{d}}\right)$ foi calculado assumindo-se $\mathrm{N}=1$, o qual foi normalizado para o teor de $\mathrm{CO}$ do solo, obtendo-se, assim, o valor de $\mathrm{K}_{\mathrm{oc}}\left(\mathrm{K}_{\mathrm{oc}}=\mathrm{K}_{\mathrm{d}} / \mathrm{CO}\right)$.

Após a coleta do sobrenadante e determinação da radioatividade no ensaio de sorção, foi iniciado o ensaio de dessorção, aproveitando-se os mesmos frascos com solo que foram utilizados no ensaio de sorção. Alíquotas de $10 \mathrm{~mL}$ da solução de $\mathrm{CaCl}_{2}$ $0,005 \mathrm{~mol} \mathrm{~L}^{-1}$, sem o pesticida, foram adicionadas aos tubos de centrífuga que continham o solo remanescente. Após o tempo de equilíbrio sob agitação (24 h) e centrifugação a 3.000 rpm por $10 \mathrm{~min}$, uma alíquota de $1 \mathrm{~mL}$ do sobrenadante foi amostrada e adicionada a $10 \mathrm{~mL}$ de solução cintiladora. A radioatividade foi determinada por ECL. Este procedimento foi repetido quatro vezes, sendo o cálculo da fração desorvida idêntico ao do ensaio de sorção. Os dados foram ajustados à equação linearizada de Freundlich.

A temperatura durante o período de agitação, tanto no experimento de sorção quanto de dessorção, foi mantida, a $25 \pm 2{ }^{\circ} \mathrm{C}$, em ambiente escuro.

\section{Cinética de sorção}

A cinética de sorção do imazaquin foi determinada pelo método "batch". Alíquotas de $10 \mathrm{~mL}$ de uma solução de imazaquin na concentração de $2,99 \mu \mathrm{mol} \mathrm{L}-1$, preparada em $\mathrm{CaCl}_{2} 0,005 \mathrm{~mol} \mathrm{~L}^{-1}$, foram aplicadas às amostras de solo $(5 \mathrm{~g}$ de solo seco). Logo após, esses tubos (40 mL) foram acondicionados em agitador horizontal, a $140 \mathrm{rpm}$. Em períodos preestabelecidos $(0 ; 0,5 ; 1 ; 3 ; 6 ; 12 ; 24$ e 48 h após a aplicação do imazaquin), alíquotas de $1 \mathrm{~mL}$ do sobrenadante foram amostradas (após centrifugação a $3.000 \mathrm{rpm}$ por $10 \mathrm{~min}$ ) e adicionadas a $10 \mathrm{~mL}$ de solução cintiladora para determinação da radioatividade por meio de ECL. A concentração do pesticida sorvida foi calculada da mesma forma que no ensaio de isotermas de sorção.

No final, o modelo de Elovich (Sparks, 1989) foi aplicado aos resultados do estudo de cinética:

$$
\frac{\mathrm{dq}}{\mathrm{dt}}=\mathrm{Xe}^{-\mathrm{Yq}}
$$

em que $\mathrm{q}=$ quantidade relativa sorvida $(\%) ; \mathrm{t}=$ tempo de equilíbrio (min); $\mathrm{X}$ e $\mathrm{Y}=$ constantes da equação, específicas ao experimento. A forma integrada desse modelo ajustada aos dados deste trabalho é:

$$
\hat{\mathrm{q}}=(1 / \mathrm{Y}) \ln (\mathrm{XY})+(1 / \mathrm{Y}) \ln \mathrm{t}
$$

em que $[(1 / \mathrm{Y} \ln (\mathrm{XY})]$ e $(1 / \mathrm{Y})$ representam o total sorvido no tempo de equilíbrio inicial (etapa rápida da cinética de sorção) e a taxa de variação da sorção de acordo com o tempo para a etapa lenta da cinética, respectivamente.

\section{RESULTADOS E DISCUSSÃO}

O modelo de Freundlich apresentou bom ajuste aos dados, constatado pelos grandes valores obtidos para $R^{2}$, maiores que 0,98 (Figura 2, Quadro 2). Como os solos apresentaram valores de $\mathrm{N}$ diferentes, o uso de $\mathrm{K}_{\mathrm{f}(\mathrm{sor}) \text {, para comparar o potencial de sorção }}$ entre os solos, não é aconselhável, pois, desta forma, as unidades do $\mathrm{K}_{\mathrm{f}(\mathrm{sor})}$ seriam diferentes para cada solo (Koskinen \& Harper, 1990). Assim, optou-se por utilizar o $K_{d}$ para avaliar a sorção do imazaquin nas amostras de solo, uma vez que os valores de $\mathrm{N}$ aproximaram-se da linearidade $(\mathrm{N}=1)$, o que reduz os erros envolvidos na estimativa do $\mathrm{K}_{\mathrm{d}}$. Ao adotar linearidade, assume-se que a sorção ocorre com baixa dependência da concentração do imazaquin na solução do solo.

A sorção de imazaquin foi baixa em todos os solos (Quadro 2), indicando fraca energia de ligação da molécula com os colóides do solo. Estes valores de $\mathrm{K}_{\mathrm{d}}$ estão coerentes com grande número de trabalhos nos quais foi avaliada a sorção do imazaquin (Renner et al., 1988; Loux et al., 1989; Regitano et al., 1997; 2000; 2005; Rocha et al., 2000, 2002). O imazaquin é um herbicida de natureza anfótera com dois grupos funcionais ionizáveis: um grupo carboxílico (ácido, 
$\mathrm{pK}_{\mathrm{a}}=3,8$ ) e um grupo quinolina (básico, $\mathrm{pK}_{\mathrm{a}}=2,0$ ) (Stougaard et al., 1990). Nos valores de pH dos solos utilizados neste experimento (Quadro 1), o imazaquin apresentava-se predominantemente na forma aniônica. Como a carga elétrica líquida dos solos

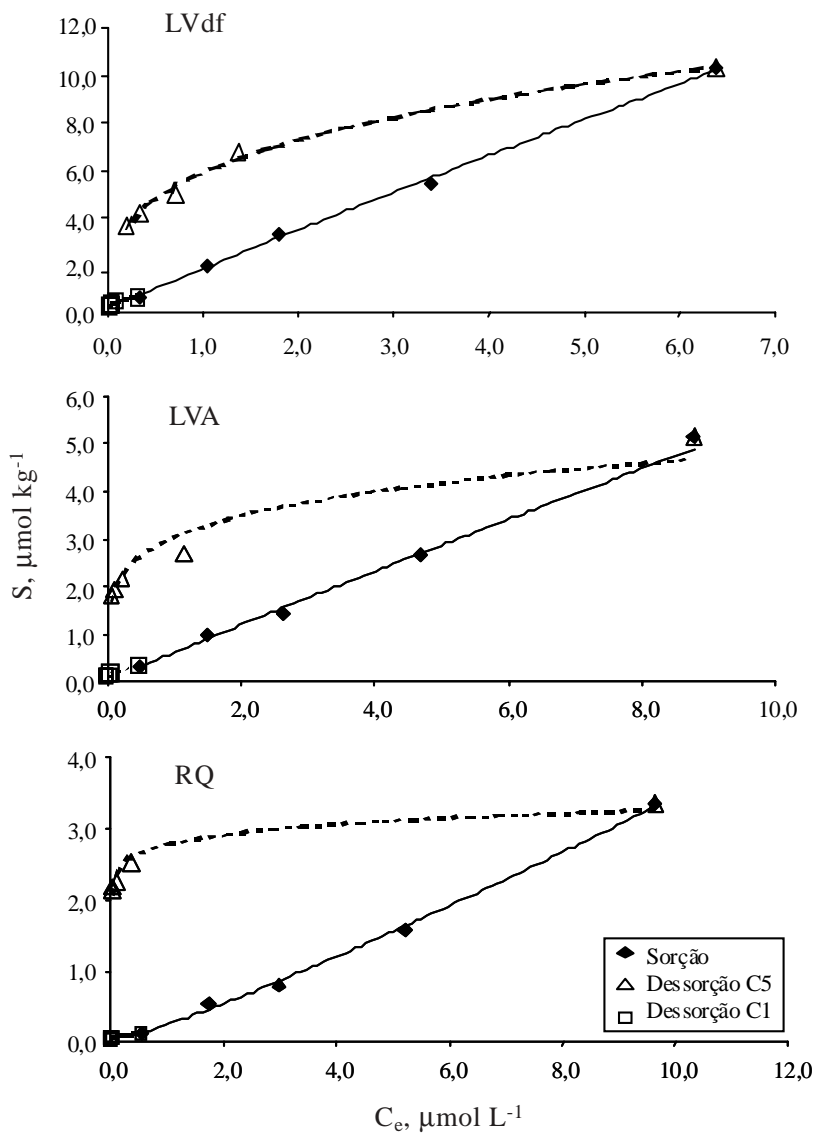

Figura 2. Isotermas de sorção (linha contínua) e dessorção (linhas tracejadas) de imazaquin em duas concentrações: $\mathrm{C} 1\left(0,67 \mu \mathrm{mol} \mathrm{L} \mathrm{L}^{-1}\right)$ e $\mathrm{C5}$ $\left(10,72 \mu \mathrm{mol} \mathrm{L} \mathrm{L}^{-1}\right) \mathrm{em}$ amostras de um Latossolo Vermelho distroférrico (LVdf), um Latossolo Vermelho-Amarelo (LVA) e um Neossolo Quartzarênico (RQ). era provavelmente negativa, evidenciada pelos valores negativos de $\Delta \mathrm{pH}\left(\mathrm{pH}_{\mathrm{KCl}}-\mathrm{pH}_{\mathrm{H}_{2} \mathrm{O}}\right)$, houve aumento da repulsão entre as moléculas de imazaquin de caráter aniônico e os sítios de carga negativa dos colóides do solo, o que concorreu para que houvesse redução na sorção.

No entanto, a repulsão devida às forças eletrostáticas não pode ser considerada o único mecanismo envolvido na baixa sorção de imazaquin em solos. A baixa afinidade do imazaquin com a matriz do solo parece estar relacionada também com a redução de sua hidrofobicidade (Reddy \& Locke, 1996). Isto se deve ao aumento da solubilidade em água do imazaquin, em razão da grande quantidade de moléculas dissociadas ( $\mathrm{pH}$ solo $>\mathrm{pK}_{\mathrm{a}}$ imazaquin). $\mathrm{O}$ aumento da solubilidade em água diminui a hidrofobicidade e, com isso, a capacidade de sorção do imazaquin nos colóides orgânicos do solo. Rocha et al. (2002) verificaram que a sorção de imazaquin em solos ácricos foi maior nas amostras subsuperficiais com baixo $\mathrm{pH}(\leq 3,4)$, quando comparada às amostras superficiais. Como nas amostras subsuperficiais o potencial elétrico do solo era positivo e o imazaquin encontrava-se predominantemente na forma neutra, condição nãofavorável ao processo de sorção, o resultado foi atribuído ao aumento da hidrofobicidade da molécula com a redução do $\mathrm{pH}$.

O coeficiente de partição octanol-água $\left(\mathrm{K}_{\mathrm{ow}}\right)$ é indicativo da hidrofobicidade da molécula. Para o imazaquin houve uma redução considerável do $\mathrm{K}_{\mathrm{ow}}$ (cem vezes menor) com a redução do valor de $\mathrm{pH}$ de 7,0 para 4,0 (Wepplo, 1991), evidenciando, mais uma vez, a influência do $\mathrm{pH}$ nas propriedades físicoquímicas do imazaquin.

Diante do exposto, verificou-se que a sorção do imazaquin no solo pareceu ser resultante da influência mútua de forças eletrostáticas (repulsivas ou atrativas) e de interações hidrofóbicas do imazaquin com a matriz do solo. Rocha et al. (2000), avaliando a sorção de imazaquin em diferentes valores de $\mathrm{pH}$ de um Latossolo, não obtiveram bom

Quadro 2. Coeficiente de Freundlich para sorção $\left(\mathbf{K}_{\mathrm{f}(\mathrm{sor})}\right)$, grau de linearidade $\left(\mathbf{N}_{\text {sor }}\right)$, coeficiente de determinação $\left(\mathbf{R}^{2}\right)$, coeficiente de sorção linear $\left(K_{d}\right)$ e coeficiente de distribuição normalizado para carbono orgânico $\left(K_{o c}\right)$, determinados em amostras de um Neossolo Quartzarênico (RQ), um Latossolo Vermelho-Amarelo (LVA) e um Latossolo Vermelho distroférrico (LVdf)

\begin{tabular}{|c|c|c|c|c|c|c|}
\hline Solo & $\mathbf{K}_{\mathbf{f}(\mathbf{s o r})}$ & $\mathbf{N}_{\text {sor }}$ & $\mathbf{R}^{2}$ & $\mathbf{K}_{\mathbf{d}}$ & $\mathbf{R}^{2}$ & $\mathbf{K}_{\mathbf{o c}}$ \\
\hline & $\left(\mu \mathrm{mol} \mathrm{kg}{ }^{-1}\right)\left(\mathrm{L} \mathrm{mol}^{-1}\right)^{\mathrm{N}}$ & & & $\mathrm{L} \mathrm{kg}^{-1}$ & & $\mathrm{~L} \mathrm{~kg}^{-1}$ \\
\hline $\mathrm{RQ}$ & $0,24(0,05)^{(1)}$ & $1,16(0,15)$ & 0,98 & $0,33(0,02)$ & 0,98 & $66,31(3,36)$ \\
\hline LVA & $0,63(0,02)$ & $0,95(0,02)$ & 0,99 & $0,58(0,03)$ & 0,99 & $52,77(3,04)$ \\
\hline LVdf & $1,83(0,02)$ & $0,93(0,03)$ & 0,99 & $1,63(0,05)$ & 0,99 & $108,82(3,60)$ \\
\hline
\end{tabular}

\footnotetext{
${ }^{(1)}$ Números entre parênteses referem-se ao desvio-padrão.
} 
ajuste do modelo proposto por Regitano et al. (1997), que estimou a sorção do imazaquin somente pela especiação de sua molécula (aniônica ou neutra) e sua partição na fração orgânica do solo, ignorando as interações com a fração mineral do solo. Os autores, a partir destes resultados, concluíram que outros fatores, como a interação da molécula (dissociada ou na forma neutra) com as cargas variáveis do Latossolo, estavam envolvidos no processo de sorção do imazaquin.

Os valores de $K_{d}$ do imazaquin foram maiores nos solos com maior teor de CO e argila (Quadro 2). Correlações positivas entre a sorção do imazaquin e teor de argila e CO no solo foram obtidas em vários estudos (Stougaard et al., 1990; Che et al., 1992; Regitano et al., 2000). De modo geral, a sorção do imazaquin está mais relacionada com o teor de CO do solo. Loux et al. (1989), avaliando a sorção de imazaquin e imazetapir, herbicidas com estruturas semelhantes do grupo das imidazolinonas, constataram dependência da sorção do imazaquin ao teor de $\mathrm{CO}$ do solo, diferentemente do imazetapir, que teve a sorção marcadamente influenciada pelo teor de argila. Estas diferenças entre as moléculas foram atribuídas à existência de um anel aromático adicional na estrutura do imazaquin, conferindo menor solubilidade em água e, conseqüentemente, maior hidrofobicidade do imazaquin, quando comparada à do imazetapir.

$\mathrm{O}$ solo LVdf apresentou o maior valor de $\mathrm{K}_{\mathrm{oc}}$, com RQ e LVA apresentando valores semelhantes (Quadro 2). Diferenças no valor de $\mathrm{K}_{\text {oc }}$ indicam mecanismos diferentes de sorção do imazaquin na matriz do solo. Como o RQ e LVA apresentam baixo teor de CO (Quadro 1), as interações hidrofóbicas da molécula com a fração orgânica podem ter, neste caso, menor influência na sorção do imazaquin que forças eletrostáticas entre os colóides do solo e o pesticida. Goetz et al. (1986) constataram que os principais componentes relacionados com a sorção do imazaquin em solo com baixo teor de $\mathrm{CO}(\leq 1 \%)$ e baixo valor de $\mathrm{pH}$ foram a caulinita e os óxidos de $\mathrm{Fe}$ e Al.

O modelo de Freundlich também ajustou satisfatoriamente as isotermas de dessorção do imazaquin, o que ficou constatado pelos altos valores de $\mathrm{R}^{2}$ obtidos (Quadro 3, Figura 2). De modo semelhante à sorção, os valores de $\mathrm{K}_{\mathrm{f}(\mathrm{des})}$ foram maiores nos solos com maiores teores de $\mathrm{CO}$ e argila (Quadro 3). Os valores de $\mathrm{K}_{\mathrm{f}(\mathrm{des})}$ foram maiores que seus respectivos $\mathrm{K}_{\mathrm{f}(\text { sor) }}$ (Quadros 2 e 3), indicando que o processo de dessorção do imazaquin envolve mecanismos com maior energia de ligação que a sorção. Esta não-singularidade ou histerese no processo de sorção-dessorção foi avaliada por meio do índice de histerese $\left(\mathrm{N}_{\text {sor }} / \mathrm{N}_{\text {des }}\right)$, que estabelece uma relação entre o grau de linearidade da sorção e o da dessorção. Quanto maior este índice, maior a histerese e maior será a irreversibilidade do processo de sorção (Seybold \& Mersie, 1996). No quadro 3, constata-se que ocorreu histerese em todos os solos, com maior intensidade no RQ.

As principais causas relacionadas com a histerese são: artifícios relacionados com o método; transformações químicas ou biológicas do composto original; frações irreversíveis ou resistentes de compostos sorvidos; impossibilidade de estabelecer equilíbrio químico entre a fase sorvida e em solução (Pignatello, 2000). Para compostos orgânicos hidrofóbicos, acredita-se que o principal mecanismo envolvido na histerese é a difusão do soluto através da estrutura da M.O. do solo, com a taxa de dessorção sendo mais lenta que a cinética de sorção (Huang et al., 2003). Esta última hipótese parece não ser válida para o caso do imazaquin nos solos estudados, haja vista que a histerese ocorreu com menor intensidade nos solos com maior teor de $\mathrm{CO}$ no solo.

Quadro 3. Coeficiente de Freundlich para dessorção $\left(\mathbf{K}_{\mathrm{f}(\mathrm{des})}\right)$, grau de linearidade $\left(\mathbf{N}_{\text {des }}\right)$, coeficiente de determinação $\left(\mathbf{R}^{2}\right)$, percentual dessorvido e índice de histerese $\left(\mathbf{N}_{\text {sor }} / \mathbf{N}_{\text {des }}\right)$, determinados em amostras de um Neossolo Quartzarênico (RQ), um Latossolo Vermelho-Amarelo (LVA) e um Latossolo Vermelho distroférrico (LVdf)

\begin{tabular}{|c|c|c|c|c|c|c|}
\hline Solo & Concentração & $\mathbf{K}_{\mathbf{f}(\text { des })}$ & $\mathbf{N}_{\text {des }}$ & $\mathbf{R}^{2}$ & Dessorvido & $\mathbf{N}_{\text {sor }} / \mathbf{N}_{\text {des }}$ \\
\hline & $\mu \mathrm{mol} \mathrm{L}-1$ & $\left(\mu \mathrm{mol} \mathrm{kg}{ }^{-1}\right)\left(\mathrm{L}_{\left.\mu \mathrm{mol}^{-1}\right)^{\mathrm{N}}}\right.$ & & & $\%$ & \\
\hline \multirow[t]{2}{*}{$\mathrm{RQ}$} & 0,67 & $0,11(0,03)^{(1)}$ & $0,11(0,01)$ & 0,96 & $47,6(3,4)$ & 10,54 \\
\hline & 10,72 & $2,57(0,22)$ & $0,05(0,01)$ & 0,89 & $28,1(5,5)$ & 23,20 \\
\hline \multirow[t]{2}{*}{ LVA } & 0,67 & $0,35(0,01)$ & $0,22(0,01)$ & 0,98 & $57,2(1,0)$ & 4,32 \\
\hline & 10,72 & $3,70(0,06)$ & $0,17(0,01)$ & 0,99 & $46,9(1,5)$ & 5,59 \\
\hline \multirow[t]{2}{*}{ LVdf } & 0,67 & $1,17(0,09)$ & $0,42(0,03)$ & 0,99 & $56,6(0,8)$ & 2,21 \\
\hline & 10,72 & $5,91(0,36)$ & $0,32(0,06)$ & 0,97 & $54,8(2,7)$ & 2,90 \\
\hline
\end{tabular}

(1) Números entre parênteses referem-se ao desvio-padrão. 
A histerese também foi maior na concentração mais alta (Quadro 3), demonstrando dependência da concentração do soluto na fase solução. Resultado também verificado em outros trabalhos (Gan et al., 1994; Berglöf et al., 2002). Gan et al. (1994) também constataram histerese na dessorção de imazetapir com valores de $\mathrm{N}$ bastante próximos dos resultados obtidos neste trabalho $\left(\mathrm{N}_{\mathrm{sor}}=0,9-1,0\right.$ e $\left.\mathrm{N}_{\mathrm{des}}=0,2-0,5\right)$.

O modelo de Elovich apresentou bom ajuste aos dados experimentais (Quadro 4, Figura 3), demonstrando que a sorção do imazaquin ocorreu em duas etapas. A primeira etapa é rápida e ocorre nos primeiros 60 minutos de contato do pesticida com o solo (Figura 3). Em razão das características do método "batch", esta etapa pode ser favorecida pela maior exposição dos sítios de equilíbrio graças à elevada relação solo:solução $(5 \mathrm{~g}$ solo: $10 \mathrm{~mL}$ solução) e à agitação. A segunda etapa foi lenta, dependente do tempo.

O LVdf apresentou o maior valor percentual de imazaquin sorvido na etapa instantânea em relação ao total aplicado $(68,49 \%)$, representado pelo valor [1/Y ln (XY)] (Quadro 4). Tal resultado pode ser atribuído ao maior teor de argila e CO do solo verificado no LVdf, o que faz com que a superfície de contato do pesticida com este solo seja maior quando comparada aos demais. Já para os demais solos, a fração sorvida instantaneamente foi menor, correspondendo a 44,46\%, no LVA, e 58,97\%, no $\mathrm{RQ}$, do imazaquin sorvido no solo. Regitano et al. (2002), avaliando a cinética de sorção do clorotalonil, também verificaram sorção significativa nos primeiros minutos de contato do pesticida com o solo. Este comportamento na cinética de sorção é bastante comum para grande número de pesticidas.

A baixa capacidade de sorção aos colóides do solo do imazaquin é confirmada neste estudo de cinética, haja vista que a quantidade total de imazaquin sorvida não foi superior a $47 \%$, no LVdf, e $11 \%$, no RQ (Quadro 4). Estes resultados corroboram os baixos valores de $\mathrm{K}_{\mathrm{d}}$ obtidos no ensaio de isotermas de sorção (Quadro 2). Como para os valores de $K_{d}$, a quantidade total de imazaquin sorvida $\left(\mathrm{S}_{\text {total }}\right)$ aumentou nos solos com maior teor de argila e CO no solo (Quadro 4). Já a quantidade de imazaquin sorvido na etapa lenta $\left(S_{\text {lenta }}\right)$ não apresentou tendência de comportamento relacionado com os componentes do solo avaliados, com o LVA apresentando a maior fração de imazaquin sorvido nesta etapa.

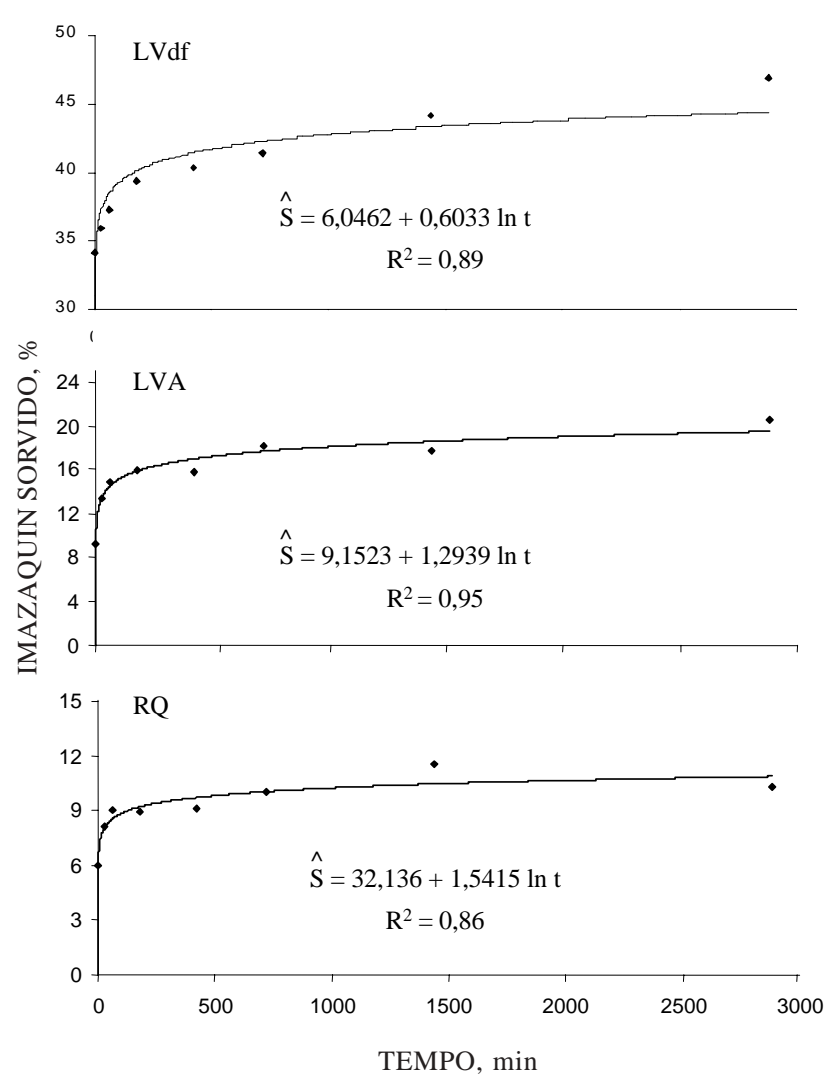

Figura 3. Modelo de Elovich aplicado aos dados de cinética de sorção de imazaquin em Latossolo Vermelho distroférrico (LVdf), Latossolo Vermelho-Amarelo (LVA) e Neossolo Quartzarênico (RQ).

Quadro 4. Parâmetros cinéticos da Equação de Elovich ajustados aos dados obtidos em amostras de Latossolo Vermelho distroférrico (LVdf), Latossolo Vermelho-Amarelo (LVA) e Neossolo Quartzarênico (RQ)

\begin{tabular}{|c|c|c|c|c|c|}
\hline \multirow{2}{*}{ Solo } & \multicolumn{3}{|c|}{ Parâmetro de Elovich } & \multirow{2}{*}{$\mathbf{S}_{\text {total }}^{(1)}$} & \multirow{2}{*}{$\mathbf{S}_{\text {lenta }}{ }^{(2)}$} \\
\hline & $1 / Y \ln (X Y)$ & $1 / \mathbf{Y}$ & $\mathbf{R}^{2}$ & & \\
\hline \multicolumn{4}{|c|}{$\%$} & \multicolumn{2}{|c|}{$\%$} \\
\hline LVdf & 32,14 & 1,5415 & 0,86 & 46,93 & 31,51 \\
\hline LVA & 9,15 & 1,2939 & 0,95 & 20,58 & 55,54 \\
\hline $\mathrm{RQ}$ & 6,05 & 0,6033 & 0,89 & 10,26 & 41,03 \\
\hline
\end{tabular}

${ }^{(1)}$ Percentagem sorvida após período de equilíbrio de $48 \mathrm{~h}\left({ }^{14} \mathrm{C}\right.$-imazaquin aplicado $\left.=100 \%\right) .{ }^{(2)}$ Percentagem sorvida na fase lenta em relação ao sorvido ${ }^{14} \mathrm{C}$-imazaquin sorvido $=100 \%$ ). 
$\mathrm{O}$ valor $(1 / \mathrm{Y})$ do modelo de Elovich expressa a velocidade de sorção do imazaquin na etapa lenta do processo de sorção. Esse valor aumentou nos solos com maior teor de argila e CO, com o LVdf apresentando o maior valor (Quadro 4). Porém, o valor (1/Y) não apresentou relação $S_{\text {lenta }}$, como pode ser constatado para o LVA, que, mesmo tendo valor de (1/Y) intermediário, apresentou o maior valor de $\mathrm{S}_{\text {lenta. Já Regitano et al. (2002) encontraram correlação }}$ positiva entre o valor $(1 / \mathrm{Y})$ e $\mathrm{S}_{\text {lenta }}$ na cinética de sorção de clorotalonil.

A sorção de compostos orgânicos na etapa lenta tem sido atribuída, principalmente, a um mecanismo de transferência de massa difusivo. A difusão é um processo aleatório que ocorre quando existe um gradiente de concentração no solo (Pignatello \& Xing, 1996).

Pela Lei de Fick, a existência de correlação entre a quantidade sorvida de um composto e o valor da raiz quadrada do tempo de equilíbrio evidencia a ocorrência de processos difusivos (Kookana et al., 1993; Regitano et al., 2002). Portanto, o bom ajuste verificado entre o imazaquin sorvido e a raiz quadrada do tempo indica que, provavelmente, a sorção do imazaquin na etapa lenta é controlada, ou, pelo menos, influenciada pela difusão do pesticida na matriz do solo (Figura 4).

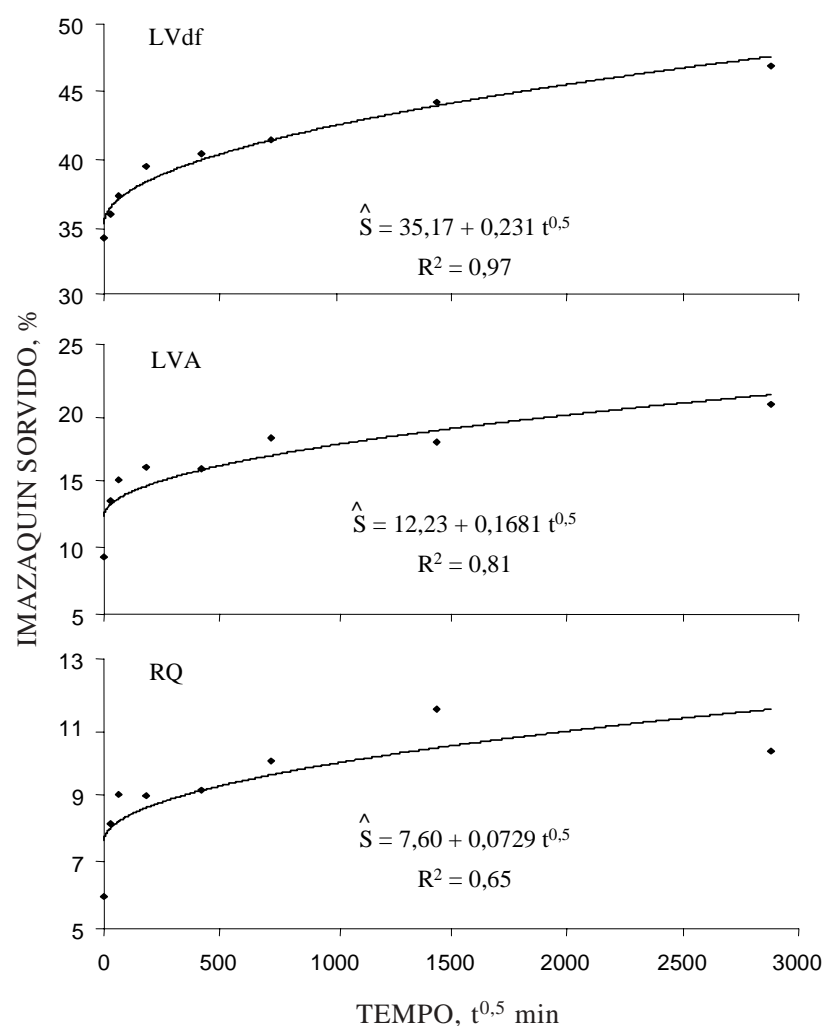

Figura 4. Percentual de imazaquin aplicado que foi sorvido em função da raiz quadrada do tempo de equilíbrio em amostras de Latossolo Vermelho distroférrico (LVdf), Latossolo Vermelho Amarelo (LVA) e Neossolo Quartzarênico (RQ).
Dentre os possíveis mecanismos de difusão, aqueles relacionados com a matéria orgânica têmse revelado extremamente importantes na sorção de compostos orgânicos, como os pesticidas. De acordo com Brusseau et al. (1991), a matéria orgânica é uma substância porosa composta de polímeros, dentro da qual o soluto pode difundir. Estas considerações, porém, são válidas para compostos orgânicos hidrofóbicos, não-iônicos. No caso do imazaquin, em razão de seu caráter anfótero, as moléculas, em sua maior parte, encontram-se dissociadas nos valores de $\mathrm{pH}$ do solo geralmente empregados nestes estudos $(\mathrm{pH}>5,0)$, reduzindo, consideravelmente, sua hidrofobicidade. Desta forma, outros mecanismos de difusão, como a difusão intrapartícula, podem estar envolvidos na difusão do imazaquin no solo. Porém, as evidências que confirmam este mecanismo de sorção não são conclusivas, sendo necessários estudos de cinética mais aprofundados para elucidar o processo de sorção na etapa lenta da reação de pesticidas no solo.

\section{CONCLUSÕES}

1. Os coeficientes de sorção do imazaquin foram baixos para todos os solos, sendo a menor sorção verificada no solo com mais baixos teores de argila e CO.

2. Os coeficientes de dessorção do imazaquin foram maiores que seus coeficientes de sorção, demonstrando ocorrer histerese na dessorção. A histerese foi verificada nos três solos avaliados.

3. A sorção do imazaquin ocorreu em duas etapas, tendo sido a segunda etapa mais lenta, influenciada por processos difusivos.

\section{LITERATURA CITADA}

BASHAM, G.W. \& LAVY, T.L. Microbial and photolytic dissipation of imazaquin persistence and mobility in three Arkansas soils. Weed Sci., 35:576-582, 1987.

BERGLÖF, T.; VAN DUNG, T. \& KYLING, H. Carbendazin sorption-desorption in Vietnamese soils. Chemosphere, 48:267-273, 2002.

BHALLA, P.N.; HACKETT, M.; HART, R.G. \& LIGNOWSKI, E.M. Imazaquin herbicide. In: SHANER, D.L. \& O'CONNOR, S.L., eds. Imidazolinones herbicides. Boca Raton, CRC Press, 1991. p.237-245.

BRUSSEAU, M.L.; JESSUP R.E. \& RAO, P.S.C. Nonequilibrium sorption of organic chemicals: Elucidation of rate-limiting processes. Environ. Sci. Technol., 25:134-142, 1991.

CAMARGO, O.A.; MONIZ, A.C.; JORGE, J.A. \& VALADARES, J.M. Métodos de análise química, mineralógica e física de solos do Instituto Agronômico de Campinas. Campinas, 1986. 94p. (Boletim Técnico, 106) 
CHE, M.; LOUX, M.M.; TRAINA, S.J. \& LOGAN, T.J. Effect of $\mathrm{pH}$ on sorption and desorption of imazaquin and imazethapyr on clays and humic acid. J. Environ. Qual., 21:698-703, 1992.

GAN, J.Y.; WEIMER, M.R. \& KOSKINEN, W.C. Sorption and desorption of imazethapyr and hydroxyimazethapyr in Minnesota soils. Weed Sci., 42:92-97, 1994.

GOETZ, A.J.; WEHTJE, G.; WALKER, R.H. \& HAJEK, B. Soil solution and mobility characterization of imazaquin. Weed Sci., 34:788-793, 1986.

HUANG W.; PENG P.; YU, Z. \& FU, J. Effects of organic matter heterogeneity on sorption and desorption of organic contaminants by soils and sediments. Appl. Geol., 18:955972, 2003.

JACKSON, M.L. Soil chemical analysis: Advanced course. Madison, American Society of Agronomy, 1969. 894p.

KOOKANA, R.S.; SCHULLER, R.D. \& AYLMORE, L.A.G. Simulation of simazine transport through soil columns using time-dependent sorption data measured under flow conditions. J. Cont. Hidrol., 14:93-115, 1993.

KOSKINEN, W.C. \& HARPER, S.S. The retention process: mechanisms. In: CHENG, H.H., ed. Pesticides in the soil environment: process, impacts, and modeling. 2.ed. Madison, SSSA. WI, 1990. p.51-77.

LOUX, M.M.; LIEBL, R.A. \& SLIFE, F.W. Availability and persistence of imazaquin, imazethapyr, and clomazone in soil. Weed Sci., 37:259-267, 1989.

PIGNATELLO, J.J. The measurement and interpretation of sorption and desorption rates for organic compounds in soil media. Adv. Agron., 69:1-73, 2000.

PIGNATELLO, J.J. \& XING, B. Mechanisms of slow sorption of organic chemicals to natural particles. Environ. Sci. Technol., 30:1-11, 1996.

RAIJ. B. van \& QUAGGIO, J.A. Métodos de análise de solos para fins de fertilidade. Campinas, IAC, 1983. 31p. (Boletim Técnico, 81)

REDDY, K.N. \& LOCKE, M.A. Imazaquin spray retention, foliar washoff and runoff losses under simulated rainfall. Pest. Sci., 48:179-187, 1996.

REGITANO, J.B.; BISCHOFF, J.B.; LEE, M.; REICHERT, J.M. \& TURCO, R.F. Retention of imazaquin in soil. Environ. Toxic. Chem., 16:397-404, 1997.
REGITANO. J.B.; ALLEONI, L.R.F.; VIDAL-TORRADO, P.; CASAGRANDE, J.C. \& TORNISIELO, V.L. Imazaquin sorption in highly weathered soils. J. Environ. Qual., 29:894$900,2000$.

REGITANO, J.B.; PRATA, F.; DIAS, N.M.; LAVORENTI, A. \& TORNISIELO, V.L. Sorção-dessorção do fungicida clorotalonil em solos com diferentes teores de matéria orgânica. R. Bras. Ci. Solo, 26:267-274, 2002.

REGITANO, J.B.; ROCHA, W.S.D. \& ALLEONI, L.R.F. Soil pH on mobility of imazaquin in Oxisols with positive balance of charges. J. Agric. Food Chem., 53:4096-4102, 2005.

RENNER, K.A.; MEGGITT, W.F. \& PENNER, D. Effect of soil $\mathrm{pH}$ on imazaquin and imazethapyr adsorption to soil and phytotoxicity to corn (Zea mays). Weed Sci., 36:78-83, 1988.

ROCHA, W.S.D.; ALLEONI, L.R.F.; REGITANO, J.B.; CASAGRANDE, J.C. \& TORNISIELLO, V.L. Influência do $\mathrm{pH}$ na sorção de imazaquin em um Latossolo Vermelho acriférrico. R. Bras. Ci. Solo, 24:649-655, 2000.

ROCHA. W.S.D.; REGITANO, J.B.; ALLEONI, L.R.F. \& TORNISIELO, V.L. Sorption of imazaquin in soils with positive balance of charges. Chemosphere, 49:263-270, 2002.

SEYBOLD, C.A. \& MERSIE, W. Adsorption and desorption of atrazine, deethylatrazine, deisopropylatrazine, hydroxyatrazine, and metolachlor in two soils from Virginia. J. Environ. Qual., 25:79-1185, 1996.

SPARKS, D.L. Kinetics of soil chemical processes. San Diego, Academic Press, 1989. 210p.

STOUGAARD, R.N.; SHEA, P.J. \& MARTIN, A.R. Effect of soil type and $\mathrm{pH}$ on adsorption, mobility, and efficacy of imazaquin and imazethapyr. Weed Sci., 38:67-73, 1990.

VETTORI, L. Métodos de análise do solo. Brasília, Ministério da Agricultura, Divisão Pedologia e Fertilidade do Solo, 1969. 24p. (Boletim Técnico, 7)

WEPPLO, P.J. Chemical and physical properties of the imidazolinones. In: SHARNER, D.L. \& O'CONNOR, S.L., eds. The imidazolinone herbicide. Boca Raton, CRC Press, 1991. p.337-452.

ZHU, H. \& SELIM, H.M. Hysteretic behaviour of metolacholor adsorption-desorption in soils. Soil Sci., 165:632-645, 2000. 